\title{
Meningeal hemangiopericytoma
}

\author{
Meltem Özdemir ${ }^{a^{*}}$,Alper Dillia ${ }^{a}$ Meltem Türk ${ }^{b}, R$ asime Pelin Kavak ${ }^{a}$ \\ a Department of Radiology, University of Health Sciences, Dışkapı Yıldırım Beyazıt Training and Research Hospital, \\ Ankara, Turkey \\ ${ }^{b}$ Department of Pathology, University of Health Sciences, Dışkapı Yıldırım Beyazıt Training and Research Hospital, \\ Ankara, Turkey
}

\section{ARTICLE INFO}

\section{Article History}

Received

Accepted

Online Published

$13 / 01 / 2020$

$06 / 02 / 2020$

$23 / 03 / 2020$

\section{* Correspondence to:}

Meltem Özdemir

Department of Radiology,

University of Health Sciences,

Ankara, Turkey

e-mail:meltemkaan99@gmail.com

\section{ABSTRACT}

Meningeal hemangiopericytomas are rare malign mesenchymal tumors accounting for less than $0.4 \%$ of all intracranial tumors. They are accepted as an aggressive form of solitary fibrous tumors and classified as World Health Organization grade II or III lesions. The clinical behavior of these tumors is highly aggressive and they are prone to local recurrence and extracranial metastasis. In clinical practice, pre-operative diagnosis of meningeal hemangiopericytomas plays a key role in treatment selection and surgical planning. However, their radiological imaging features can be almost indistinguishable from those of meningiomas. Here, we aimed to present a 46-year-old male with anaplastic hemangiopericytoma and to emphasize the distinctive radiological features specific to hemangiopericytoma.

\section{Keywords:}

Intracranial tumor

Meningeal hemangiopericytoma

Meningioma

(C) $2020 \mathrm{OMU}$

Surgery

\section{Introduction}

Meningeal hemangiopericytomas (HPC) are rare malign mesenchymal tumors accounting for less than $0.4 \%$ of all intracranial tumors. They mostly occur in younger adults with a mean presentation age of 3842 years. Approximately $10 \%$ of all HPCs occurs in children and show a slight male preference (Smith et al., 2014). While HPC is widely thought to develop from pericytes surrounding capillaries, the exact origin of the tumor remains controversial. Currently, it is accepted as an aggressive form of solitary fibrous tumors and classified as World Health Organization (WHO) grade II or III lesions. The WHO grade III encompasses the anaplastic HPCs (Giannini et al., 2007). HPCs usually present with headache, seizures, visual impairment and motor weakness. And in imaging studies, they appear as a locally aggressive, bulky dural mass. However, HPCs are very similar in terms of radiological features to meningiomas, which are much more common than they are. Therefore, HPCs are often indistinguishable from meningiomas in imaging studies and have a high rate of misdiagnosis (Bouvier et al., 2012; Liu et al., 2013; Smith et al., 2014). Here, we aimed to present a 46-year-old male with anaplastic HPS and to emphasize the distinctive radiological features specific to HPS. 


\section{Case}

A 46-year-old male patient presented with headache that had been present for the last six months and had increased significantly in the last two weeks. He also reported weakness and numbness in his right arm for the past few months. Neurological examination revealed loss of strength in the patient's right arm compared to the left. Head computed tomography (CT) revealed a bulky mass in the right temporal lobe which caused erosion at the adjacent skull (Fig. 1). On brain magnetic resonance imaging (MRI), the lesion appeared as a lobulated extraaxial mass measuring 54 × 32 × $53 \mathrm{~mm}$ (AP x T x CC). It was a heterogeneous lesion with central necrotic areas and perilesional edema. Due to the mass effect of the lesion, the adjacent cortical sulci as well as the lateral ventricle were effaced and the midline structures were pushed towards the contralateral side (Fig. 2). The mass was hypointense in T1-weighted, T2-weighted and FLAIR images. Diffusion-weighted images and ADC mapping showed intermediate restriction of diffusion within the mass (Fig. 3). An intense heterogeneous enhancement following gadolinium administration was recorded. The mass showed a relatively narrow base of dural attachment and dural tail sign (Fig. 4). Digital subtraction angiography (DSA) demonstrated the arterial supply from the external carotid artery and rich vascularization within the mass. In addition, a characteristic "fluffy" stain was noted within the lesion (Fig. 5).

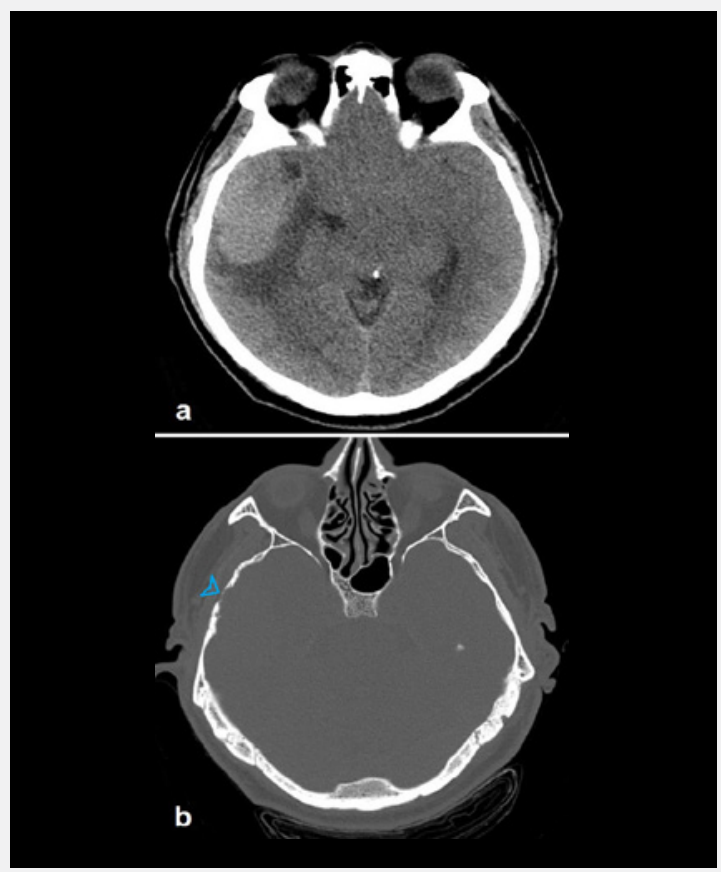

Fig. 1. Axial computed tomography sections through parenchyme (a) and bone (b) windows show a large mass located in the right temporal lobe causing erosion at the adjacent skull (arrowhead).

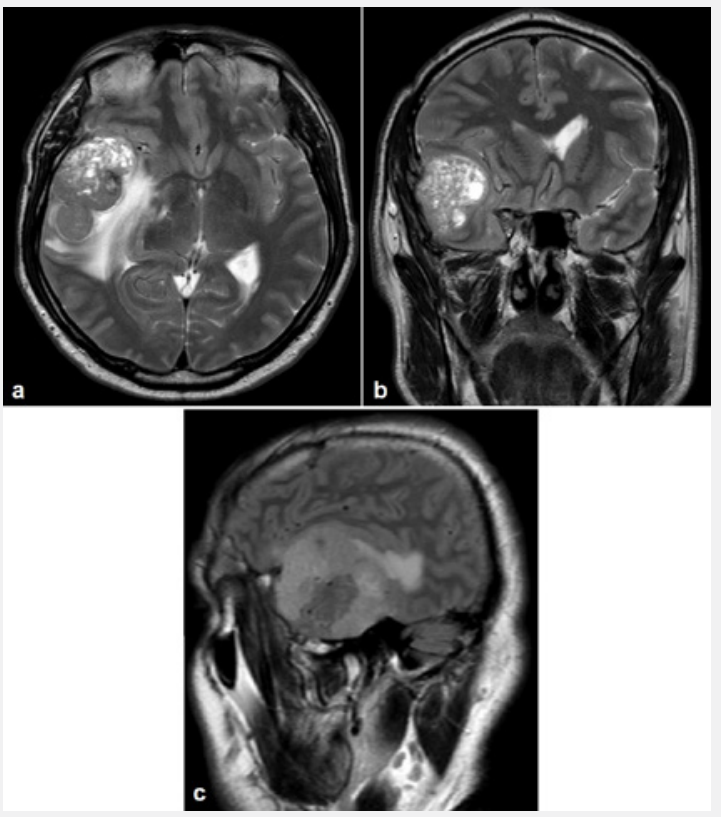

Fig. 2. Axial (a), coronal (b) and sagittal (c) T2-weighted brain magnetic resonance images show a lobulated, extra-axial, heterogeneous mass with central necrotic areas and perilesional edema in the right temporal lobe. Due to the mass effect of the lesion, the adjacent cortical sulci as well as the right lateral ventricle are effaced and the midline structures are pushed towards the contralateral side.

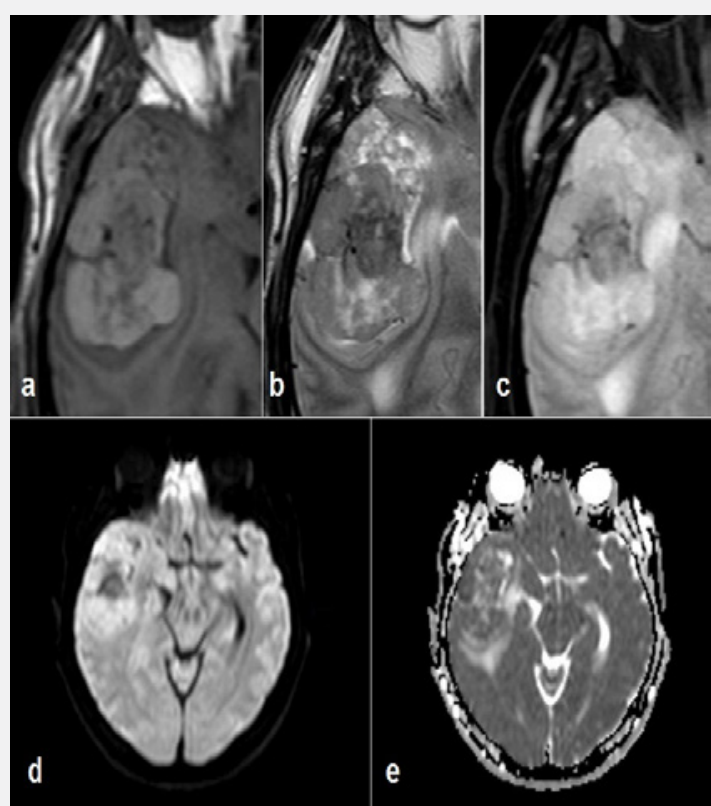

Fig. 3. The mass is isointense in T1-weighted (a), T2weighted (b) and FLAIR (c) images. Diffusionweighted image (d) and ADC mapping (e) show intermediate restriction of diffusion within the mass. 


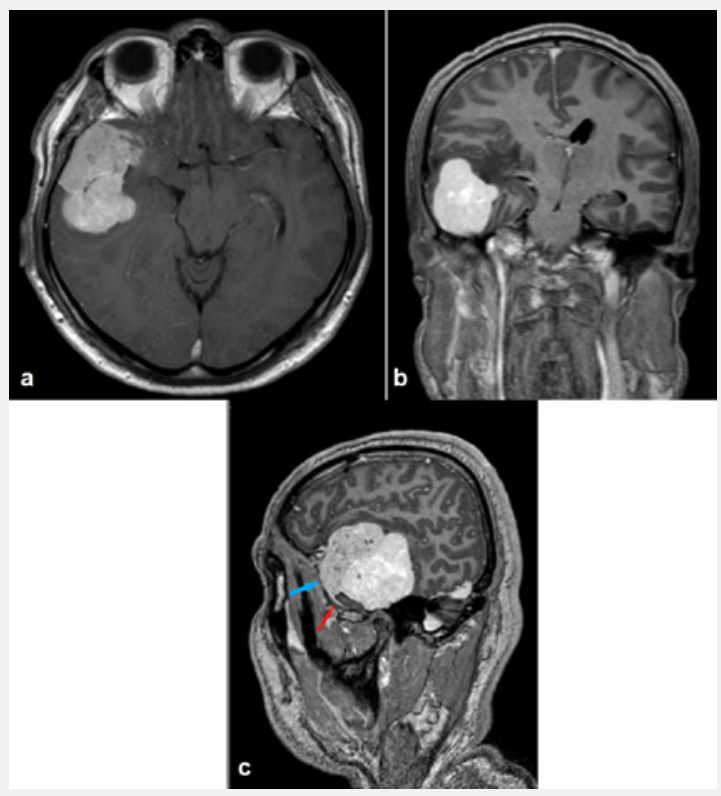

Fig. 4. Post-contrast axial (a), coronal (b) and sagittal (c) T1-weighted images show an intense heterogeneous enhancement of the mass. Note the mass shows a relatively narrow base of dural attachment (blue arrow) and dural tail sign (red arrow).

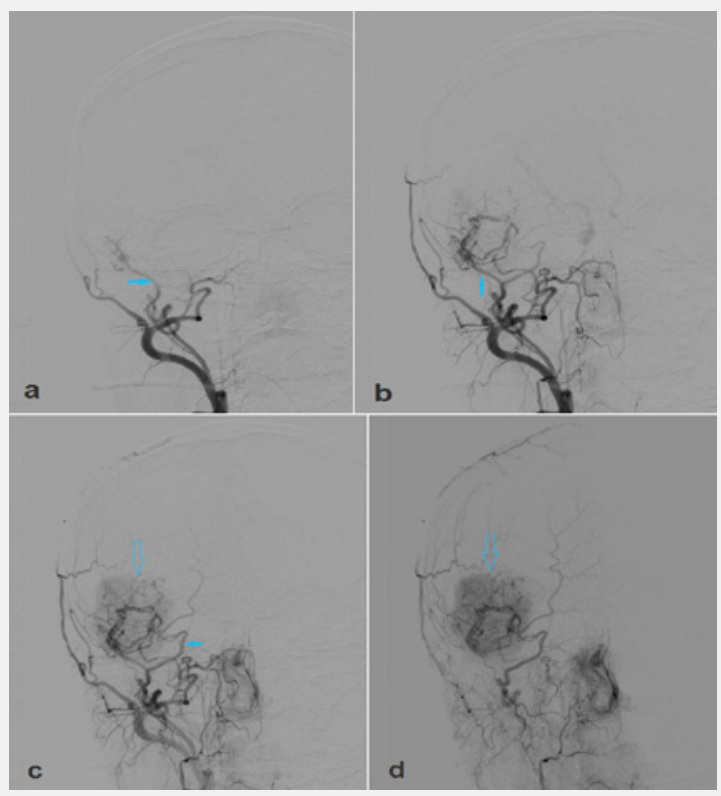

Fig. 5. Consecutive digital subtraction angiography images demonstrate the arterial supply from the deep temporal artery branches of the right maxillary artery (solid arrows). Note the rich vascularization and fluffy stain within the mass (oper arrows).

Based on the imaging findings, the lesion was diagnosed as meningeal hemangiopericytoma. A comprehensive imaging study was then performed to exclude a possible metastasis and no metastatic focus was detected. Following the pre-operative catheter embolization, en bloc resection of the mass was performed. On pathological evaluation, the tumor exhibited high mitotic activity with spindle character occasionally showing a storiform pattern. Immunohistochemical preparations for CD34 were focally positive (Fig. 6). Pathological examination confirmed the pre-operative diagnosis and the lesion was categorized as WHO grade III HPC. Adjuvant radiotherapy was planned to prevent recurrence.

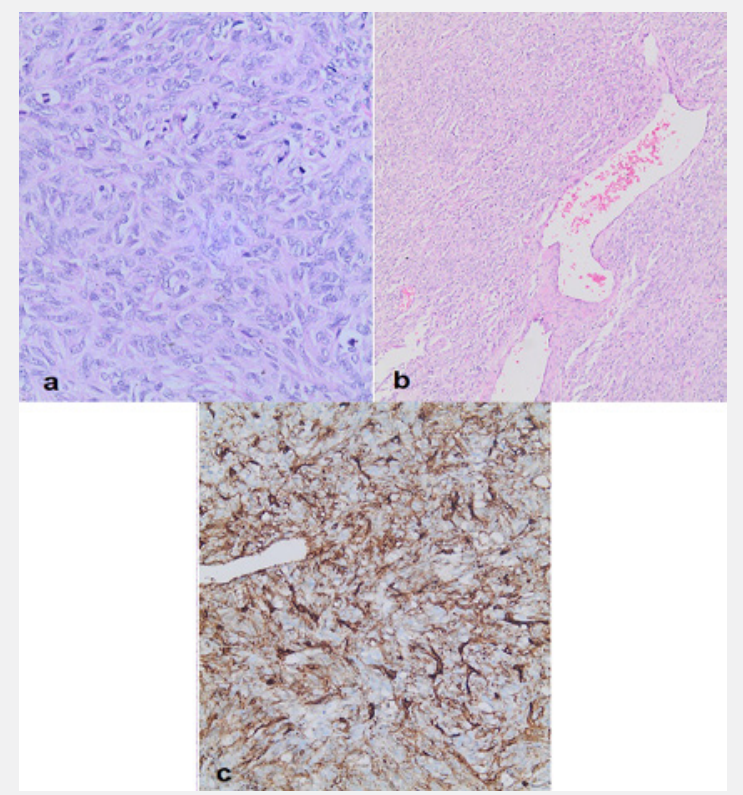

Fig. 6. (a) (H\&E 100x) The tumor exhibits high mitotic activity with spindle character occasionally showing a storiform pattern. (b) (H\&E 100x) In some places, fine vascular structures in the form of antlers were recorded. (c) (IHC 200x) There are patchy areas of CD 34 positivity in tumor cells.

\section{Discussion}

While HPC is considered a mesenchymal tumor, it exhibits different clinical behaviors, histological classification and immunohistochemical features compared to other primary mesenchymal tumors such as meningioma. Therefore, according to the WHO classification of central nervous system tumors, HPC is categorized as "mesenchymal, non-meningothelial tumors". HPC is much more aggressive and has a high recurrence rate compared to meningioma (Giannini et al., 2007). In clinical practice, pre-operative diagnosis of HPCs plays a key role in treatment selection and surgical planning. However, pre-operative diagnosis of HPC is not always easy. In most cases, the mass exhibits very similar imaging features to the meningioma, making it extremely difficult for the radiologist to make differential diagnosis (Liu et al., 2013; Smith et al., 2014). 
We presented a patient with HPC showing a narrow dural attachment, contour lobulation and skull erosion, suggestive of a HPC rather than a meningioma which is characterized by a broad dural attachment, regular contours and hyperostosis at the adjacent skull. HPCs almost always present as a large and locally aggressive dural mass causing skull destruction and frequently extending through the skull vault. However, meningiomas are characterized by overgrowth of the adjoining bone, which is called "hyperostosis" (Bouvier et al., 2012; Smith et al., 2014). Further, the mass lesion we currently present depicted a "fluffy" stain in DSA unlike a meningioma that typically exhibits a "sunburst" stain (Liu et al., 2013). Other findings that excluded the diagnosis of meningioma in our case were extensive perilesional edema and intratumoral necrosis including old bleeding foci and cystic sites. Intratumoral necrosis and peritumoral edema are striking findings supporting HPC, especially the anaplastic form, as we have now presented (Giannini et al., 2007; Bouvier et al., 2012; Liu et al., 2013; Smith et al., 2014).

In addition to imaging features, there are some clinical clues that can help distinguish HPC and meningiomas. First, the average age of presentation of HPCs (mean, 38-42) is lower than that of meningiomas which mostly manifest themselves at the early fifties. Second, the interval between initial symptoms and the diagnosis of HPC (mean, 4 months) is significantly shorter than that of meningiomas which is 1-2 years on average. Third, the clinical behavior of HPCs is more aggressive than that of meningiomas. And different from meningiomas, HPCs have a strong tendency to local recurrence and extracranial metastasis (Liu et al., 2013; Abdollahi et al., 2016).

The presentation symptoms of our case were headache and weakness in the right arm. According to the previously reported cases, the presentation of the tumor depends on the location of the lesion, but the symptoms usually include headache, seizures, visual impairment and motor weakness. Headache is reported to be the most common symptom in patients with HPC (Liu et al., 2013; Abdollahi et al., 2016; Tatarlı et al., 2016). The treatment of HPC consists of surgical resection and post-operative adjuvant radiotherapy. Since they are highly vascular tumors pre-operative embolization is highly recommended in these cases (Keser et al., 2014; Smith et al., 2014; Tatarlı et al., 2016).

Meningeal HPCs are rare malign mesenchymal tumors of which at times the radiological imaging features can be almost indistinguishable from those of meningiomas. Knowledge of the distinctive imaging features as well as characteristic clinical clues can help distinguish HPS and meningioma.

Informed consent: Informed consent for publication was obtained from the patient.

Conflict of Interest: The authors declare that they have no conflict of interest.

Funding: This research did not receive any specific grant from funding agencies in the public, commercial, or not-for-profit sectors.

\section{REFERENCES}

Abdollahi, A., Abdollahpouri, R., Tavangar, S.M., 2016. Meningeal hemangiopericytoma in 33-year-old female; a case report. Iran. J. Pathol. 11, 281-285.

Bouvier, C., Métellus, P., de Paula, A.M., Vasiljevic, A., Jouvet, A., Guyotat, J., Mokhtari, K., Varlet, P., Dufour, H., FigarellaBranger, D., 2012. Solitary fibrous tumors and hemangiopericytomas of the meninges: Overlapping pathological features and common prognostic factors suggest the same spectrum of tumors. Brain. Pathol. 22, 511-521.

Giannini, C., Rushing, E.J., Hainfellner, J.A., 2007. Haeman giopericytoma. In: Louis DN, Ohagaki H, Wiestler OD, Cavanees WK, eds. WHO classification of tumours of the central nervous system. Lyon, France: IARC, 178-180.

Keser, N., Çelikoğlu, E., Iş, M., Tutkan, I., Aydın, I. H., Kevenk, A.U., Somay, A., 2014. Intracranial hemangiopericytoma: A case report. J. Ist. Faculty. Med. 77, 8-11.

Liu, G., Chen, Z.Y., Ma, L., Lou, X., Li, SJ., Wang, Y.L., 2013. Intracranial hemangiopericytoma: MR imaging findings and diagnostic usefulness of minimum ADC values. J. Magn. Reson. Imaging. 38, 1146-1151.

Smith, A.B., Horkanyne-Szakaly, I., Schroeder, JW., 2014. From the radiologic pathology archives: Mass lesions of the dura: Beyond meningioma-radiologic-pathologic correlation. Radiographics. 34, 295-312.

Tatarlı, N., Gergin, Y.E., Özdoğan, S., Yavuzer, D., Tiryaki, M., Hiçdönmez, T., 2016. Supratentorial hemangiopericytoma: Case report. J. Kartal. TR. 27, 142-144. 